\title{
Notas para una cartografía oral del cielo entre los tobas del oeste formoseño
}

Notes pour une cartographie orale du ciel chez les Tobas du nord-ouest de Formosa

Notes towards an Oral Cartography of the Sky among the Toba of Western Formosa

\section{Cecilia Paula Gómez}

\section{OpenEdition}

Journals

Edición electrónica

URL: http://journals.openedition.org/bifea/8448

DOI: $10.4000 /$ bifea. 8448

ISSN: 2076-5827

\section{Editor}

Institut Français d'Études Andines

\section{Edición impresa}

Fecha de publicación: 1 agosto 2017

Paginación: 311-329

ISSN: 0303-7495

\section{Referencia electrónica}

Cecilia Paula Gómez, « Notas para una cartografía oral del cielo entre los tobas del oeste formoseño », Bulletin de l'Institut français d'études andines [En línea], 46 (2) | 2017, Publicado el 08 agosto 2017 consultado el 05 noviembre 2020. URL : http://journals.openedition.org/bifea/8448 ; DOI : https:// doi.org/10.4000/bifea.8448

\section{(c) $(1) \odot$}

Les contenus du Bulletin de l'Institut français d'études andines sont mis à disposition selon les termes de la licence Creative Commons Attribution - Pas d'Utilisation Commerciale - Pas de Modification 4.0 International. 


\title{
Notas para una cartografía oral del cielo entre los tobas del oeste formoseño
}

\author{
Cecilia Paula Gómez*
}

\begin{abstract}
Resumen
Este artículo propone un estudio de la visión toba del cielo: los objetos celestes, asterismos y aquellos fenómenos que desde la óptica nativa se relacionan significativamente con el firmamento. Esta interpretación de lo celeste se relaciona a su vez con la cosmología, la mitología y la organización social. Parte fundamental de esta conexión es la diferencia entre los seres celestes masculinos, quienes cumplen con las etapas del ciclo vital, y los femeninos, que parecen cristalizados en una condición inalterable.
\end{abstract}

Palabras clave: etnoastronomía, cielo, tobas, asterismos, Gran Chaco

\section{Notes pour une cartographie orale du ciel chez les Tobas du nord- ouest de Formosa}

\section{Résumé}

Cet article propose une étude de la vision toba du ciel : les objets célestes, les astérismes et les phénomènes qui selon l'optique autochtone sont en relation avec le firmament de façon significative. Cette interprétation du ciel correspond à la cosmologie, la mythologie et l'organisation sociale des Tobas. Une partie fondamentale de cette connexion tient à la différence entre les êtres célestes masculins, qui accomplissent les étapes du cycle vital, et les féminins, qui paraissent cristallisés dans une condition inaltérable.

Mots-clés : ethnoastronomie, ciel, Tobas, astérismes, Grand Chaco 


\title{
Notes towards an Oral Cartography of the Sky among the Toba of Western Formosa
}

\begin{abstract}
The purpose of this article is to study the Toba vision of the sky: celestial objects, asterisms, and those phenomena that, from a native perspective, are significantly related to the sky. This interpretation of the celestial abode is also related to their cosmology, mythology, and social organisation. A key part of this connection is the difference between male celestial beings, which fulfil the life-cycle stages, and female celestial beings, which seem to be crystalized in an unchanging condition.
\end{abstract}

Keywords: Ethnoastronomy, sky, Toba Indians, asterisms, Gran Chaco

\section{INTRODUCCIÓN}

Los indígenas chaqueños no perciben el cielo como un espacio disociado del medioambiente, de la estacionalidad anual, de la meteorología o aun de la propia vida social. Combinando la investigación de las percepciones tobas de lo celeste con el conocimiento básico de la astronomía de posición, el presente trabajo forma parte de un estudio más amplio que comenzó a fines del año 2004. Busca documentar antropológica e históricamente la percepción cultural del cielo de los indígenas chaqueños y, en particular, de los tobas del oeste formoseño, su relación con la mitología y la organización sociopolítica nativa.

En el Chaco Central, diversos reportes han descrito con mayor o menor detalle las interpretaciones indígenas del cielo y sus asterismos1. Uno de los primeros autores en dedicarse al tema fue Erland Nordenskiöld (2002 [1912]: 48, 101). Al descubrir cómo los indígenas chaqueños miden el tiempo, el sueco especifica brevemente las ideas chorotes sobre los eclipses lunares y los meteoros; detalla también la relación entre las Pléyades y la época de cosecha, mostrando cómo tanto los nivaclés (chulupíes o ashluslays) como los chorotes se sirven de la luna para medir lapsos temporales breves. Posteriormente, Roberto Lehmann-Nitsche publicó los primeros trabajos dedicados específicamente a la descripción chaqueña del cielo (Lehmann-Nitsche, 1923a; 1923b; 1924a; 1924b; 1924c; 1924-1925; 1927). Enrique Palavecino (1933), por su parte, hace alguna mención al sol y a su utilidad a la hora de medir distancias. Pero hay que esperar a mediados del siglo XX para que Alfred Métraux (1946b) publique varios mitos tobas y pilagás que revelan sus ideas respecto del sol, la luna y otros cuerpos celestes (1946b:19-49; 1944; 1946a; 1973).

Más allá de la región específica del Pilcomayo, algunas veces es bastante dificultoso distinguir con certeza a qué parcialidad chaqueña corresponde cada una de las observaciones. Por ejemplo, Storm (1892) explica que los indígenas cuentan los

1 Entiendo por «asterismos» a las constelaciones pero, a la vez, a todas aquellas partes del firmamento significativas para los indígenas (p. ej. una zona oscura o brillante de la vía láctea). 
meses y las semanas con la ayuda de los períodos lunares y que el sol les sirve como reloj. Aráoz cuenta también cómo un lenguaraz del río Bermejo, probablemente toba, medía la distancia entre un sitio y otro contando lunas (Aráoz, 1884: 128). Los misioneros jesuitas que estuvieron en el Chaco en el siglo XVIII como Dobrizhoffer (1968 [1783]), Lozano (1989 [1733]) o Paucke (2010 [1749-1767]) dejaron importante información que, aunque no pertenezca estrictamente a la zona aquí referida, sirve para iluminar y entender mejor la casuística toba.

Es sobre todo desde principios y hasta mediados del siglo XX que, finalmente, comienza a describirse de forma más o menos sistemática la visión indígena del cielo. Nuestras referencias más precisas se limitan a principios del siglo XX, porque en la zona del Pilcomayo medio, donde el frente colonizador se instala de forma definitiva recién en 1911, las fuentes no tienen gran profundidad temporal, al contrario de lo que sucede en las periferias del Chaco, o en regiones aledañas como los Andes o la Chiquitanía (Rostagno, 1969 [1911]; Gordillo, 2001; Combès et al., 2009; Bossert et al., 2011). Por esta razón, a la vez, es solo hacia fines del siglo XX que el problema etnoastronómico como tal cobra su dimensión actual2.

\section{LOS TOBAS DEL PILCOMAYO MEDIO}

También Ilamados «tobas del oeste formoseño», «tobas-pilagá», «tobas de Sombrero Negro» o «tobas ñachilamole'k», los tobas del Pilcomayo medio habitan aproximadamente a 60 kilómetros de la ciudad Ingeniero Juárez, al noroeste de la actual provincia de Formosa (Argentina), muy cerca de la frontera con Paraguay. Por lo tanto, los poblados rurales se encuentran muy cercanos al río Pilcomayo: Vaca Perdida, La Rinconada, El Churcal, La Mocha, 6 de Abril, Tres Yuchanes, Pozo Ramón, Isla García, La Madrugada, Pozo Charata, El Breal, La Bolsa, Laguna Cansino, Quebrachito, Barrio Méndez, etc. Actualmente, los tobas habitan también los alrededores de la ciudad de Ingeniero Juárez, en un barrio periurbano Ilamado Barrio Toba (Arenas, 2003; 2012; Arenas \& Porini, 2009; Mendoza, 2002; Gordillo, 2005).

Está documentado que el idioma de este grupo es más cercano al de los pilagás y tobas occidentales que a la lengua hablada por los tobas orientales (Métraux, 1937a; Tebboth, 1943; Arenas, 2003; Carpio, 2012). Por otra parte, el grupo se caracteriza también por estar rodeado por poblados wichís, indígenas de otro tronco lingüístico, con lo cual pueden notarse consonancias lingüísticas para (al menos) el sistema de números (Carpio, 2009). También se puede mencionar al respecto la antigua organización social en bandas nombradas, excepcional en otras unidades étnicas denominadas «tobas» pero frecuente entre los wichís, lo cual hace pensar en un juego fluido de préstamos culturales (Braunstein, 19881989; Mendoza, 2002).

2 Véanse por ej. los estudios de Braunstein, 1989; Cordeu, 1969-1970; 1977; 2000; Giménez Benítez et al., 2002; 2006; López, 2009; Idoyaga Molina, 1989; 2008; Dasso, 1989; Ruiz Moras, 2000; Terán, 1998; 2000; Palavecino, 1969-1970. 
A las relaciones intertribales hay que añadir el contacto paulatino de los tobas con la sociedad envolvente. Así, a comienzos del siglo XX, se vieron progresivamente acorralados por los colonos criollos $y$, en una zona fronteriza, por los ejércitos argentino, boliviano y paraguayo. Los territorios que solían recorrer, que incluían tierras del otro lado del Pilcomayo, fueron cada vez más limitados. Además, el trabajo asalariado comenzó en los ingenios, lo que también implicó cambios en la organización social. En ese contexto, los indígenas decidieron solicitar una misión anglicana que, finalmente, se instaló entre ellos en octubre de 1930. El asentamiento de los misioneros anglicanos en Sudamérica puede rastrearse desde los intentos de Allen Gardiner por evangelizar a los indígenas de este continente. Gardiner arribó a Sudamérica en 1838 e intentó crear misiones tanto en el sur como en el Chaco. En 1888 logra establecer una misión entre los indígenas lengua-mascoy, Makthalawaya. Poco a poco los misioneros logran incursionar en el Chaco argentino apoyados por la familia Leach, dueña de ingenios azucareros, y prosiguen fundando misiones. Los tobas tenían noticias de estos asentamientos anglicanos y, a partir de 1928, comienzaron a solicitar una misión para ellos en Misión San Andrés: hicieron el pedido varias veces hasta que el misionero anglicano Alfred Leake se instaló entre ellos en la fecha mencionada arriba. La misión propició una nueva dinámica social, un nuevo circuito económico y cultural entre los tobas. Una de las preocupaciones principales de los misioneros fue la educación. Por otro lado, el tratamiento médico que brindaban fue de gran importancia y fundó buena parte de la confianza que los indígenas les tenían; confiaban en sus poderes de curación. Los misioneros de la South American Missionary Society se transformaron en protectores y asumieron progresivamente muchas de las funciones tradicionales del haliaGa 'nek o líder. La misión El Toba funcionó hasta que la inundación de 19751976 acabó con la ribera del río Pilcomayo. Por sugerencia de un misionero, los tobas se mudaron hacia un lugar más alto y seguro, Ilamado Vaca Perdida, en torno del cual fueron surgiendo los asentamientos actuales. La impronta misionera en el plano celeste, por ende, aún puede notarse.

\section{HACIA UNA CARTOGRAFÍA ORAL DEL CIELO}

El proyecto más general en el que se inscribe este trabajo comenzó durante el mes de febrero, en una etapa muy calurosa que los tobas engloban en su ciclo anual bajo la categoría de no 'laGa y que consideran poco propicia para observar el cielo nocturno. Dicen que «el cielo está más lejos» y por lo tanto no se ve bien. Por eso los colaboradores recomendaron regresar durante la parte más fría del año, nakabia'Ga, durante los meses de junio y julio, cuando el cielo estaría «más cerca» y se podría ver mejor: «la oscuridad es más perfecta (...) muy bajito están las estrellas». Pero esta proximidad del firmamento, a la vez, tiene consecuencias: produce un frío intenso. El frío se asocia con la cercanía del cielo o pi'yem. Para entender el vínculo podemos recordar el mito de la mujerestrella, muy generalizado en el área chaqueña3. En él se describe el cielo como

3 Varias versiones tobas del relato fueron publicadas por Wilbert \& Simoneau, eds. (1982b: 51-61). Hay también numerosas versiones en otros grupos étnicos de la zona (Wilbert \& Simoneau, eds., 
un espacio helado, donde hay fuegos que explotan y no calientan, poblado de seres peligrosos que pueden bajar a la tierra; en suma, es visto como un espacio poco apto para la vida humana. De hecho, las mujeres-estrella pueden bajar a la tierra, pero el hombre que intenta hacer lo inverso muere. Es decir que, al menos en principio, el cielo parece un ámbito vedado al hombre, salvo que se trate de un shamán pioGo 'nak. Quienes realmente saben cómo es el cielo son los shamanes, que son los únicos capacitados para viajar por él4. Algunas de las personas que describen el cielo se basan en informaciones brindadas por ellos y, en los últimos tiempos, muchas veces respaldan sus dichos en explicaciones construidas a partir de la hermenéutica bíblica 5 . Para no sesgar la información, al reflejar solamente la opinión de los que podrían ser considerados especialistas religiosos, hemos intentado no recurrir solo a los pioGónak para interpretar el plano celeste sino a la vez a ancianos, jóvenes y mujeres. De esta manera pudimos documentar los objetos celestes más relevantes en distintas etapas del ciclo anual, combinando la observación de campo con punteros láser y programas informáticos (Stellarium).

Antes de comenzar con la descripción de los asterismos, debemos subrayar que las lecturas de lo celeste son un tópico abierto al juego de las interpretaciones. La ubicación y denominación de algunos de ellos pueden provocar discrepancias individuales y/o grupales. Sin embargo, el saber etnoastronómico es un conocimiento que los tobas más ancianos pretenden mantener vivo en la memoria de los más jóvenes; por eso se preocupan de forma explícita para que «los estudios de los antiguos» no caigan en el olvido.

\section{ESTRELLAS, ASTERISMOS Y OBJETOS CELESTES}

El cielo o pi'yem es considerado un ámbito femenino, del cual, según el mito, provienen las mujeres (ia 'wo) que bajan a la tierra. Aquellas estrellas (waqachi'ñi) que no forman parte de ningún asterismo son aquellas que no descienden a la tierra, o las que lograron regresar hasta su lugar de origen sin ser atrapadas por los hombres/animales primigenios que, antiguamente, poblaban la tierra. El mito que cuenta el origen celestial de las mujeres está muy difundido en el área chaqueña ${ }^{6}$. Narra la forma en que las mujeres primordiales bajaban del cielo para comer las

1982a: 48; 1985: 254-268; 1989: 57-61; Dasso, 1989: 32; Braunstein, 1993: 41-46; Barúa \& Dasso, 1999: 261-262; Palmer, 2005: 288-296; Gómez, 2007: 97-116; 2009: 205-219; Idoyaga Molina, 2008: 91-104).

4 Sobre el chamanismo chaqueño y toba en particular, véase Miller, 1975.

5 Aunque hasta el día de hoy los tobas del oeste formoseño no cuentan con una traducción íntegra de la Biblia en su propio idioma, realizan una interpretación de la misma guiada por los estudios que los pastores tobas de la comunidad hicieron con los anglicanos. A ello se suman las propias reflexiones, tanto de los pastores tobas, como de los fieles. Para indagar más sobre el tema, véase Gómez, 2008.

6 Ver p. ej. Lehmann-Nitsche (1923b: 284-285); Métraux (1946b: 102-107); Cordeu (1969-1970: 143-148); Palavecino (1969-1970: 185-186); Miller (1977: 323-325); Wilbert \& Simoneau (1982a: 67-77; 1982b: 101-143; 1985: 29-38; 1989: 130-155); Pérez Diez (1983: 134-169); Idoyaga Molina (1986: 165-174); Barúa \& Dasso (1999: 253-257). 
provisiones de los hombres/animales primigenios con sus vaginas dentadas. Pero un día los hombres lograron atraparlas; las mujeres que no pudieron regresar al cielo y seguir siendo estrellas comenzaron a poblar la tierra y a tener relaciones sexuales con los hombres/animales. Para que comenzaran a nacer los hombres y mujeres actuales, wayaGaka'lachigi (el Zorro) se encargó de romper los dientes que tenían las mujeres en la vagina (Gómez, 2010).

Sin embargo, en el ámbito celeste también hay asterismos sin connotaciones femeninas tan marcadas: uno de ellos es NeGeonak neqow`ka, la «Tierra Revuelta por el Conejo» que está formado por las Nubes de Magallanes. La Nube de Magallanes más grande era llamada «madriguera del conejo», en tanto que su homóloga más pequeña era el «Campo de Juego de los Conejos». Representada por tres estrellas de la constelación Can Mayor, también se reportaron las cercanas «Huellas de los Conejos» (Métraux, 1946b: 21). El nombre de Nalonaka’te o «Las Palomitas» también aparece refiriendo tanto a las Columbina Picui como a las Columbina talpacoti, asociadas a su vez con ciertas aves que vuelan en parejas: por ese motivo, una de las estrellas señaladas es Theta uno $(\theta 1)$ y la otra Theta dos $(\theta 2)$ de la constelación de Tauro. Sin embargo, los tobas también asocian ese nombre con otras estrellas cercanas a la Cruz del Sur. Reconocen también a La Cruz del Sur y, al igual que los criollos, aunque en su idioma, la llaman So'doso (cruz). Por su parte, el ave llamada Qo'towoik (Cathartes aura) es representada por la estrella lambda $(\lambda)$ de la constelación Orión. Se dice, asimismo, que existían unas estrellas que representaban a los Perros ('Piog), ubicadas cerca de la Cruz del Sur y de la Vía Láctea. Finalmente, Ilaman a las estrellas fugaces waqachi’ ñi latek, por unos hongos de monte (Calvatia aff.cyathiformis, Calvatia fragilis, Mycenastrum corium, Tulostoma y Vascellum pampeanum) cuya traducción sería «excremento de estrella», los cuales sirven para sanar heridas (Arenas, 1992-1993: 99).

Venus es reconocida como una estrella, y cuando los tobas la ven junto a la luna afirman que es «su esposa». Para referirse a ella utilizan dos nombres distintos: si la ven al amanecer (lucero matutino), la llaman 'Nichigi; si la observan al atardecer (lucero vespertino), la llaman Pota'kae. Se asocia directamente con el comienzo y el fin de las labores cotidianas: 'Nichigi indica la hora de levantarse e ir a trabajar y Pota'kae indica por la tarde el momento de terminar las labores. Por otro lado, se asocia a 'Nichigi con el mito de la mujer-estrella y el relato muestra algunas similitudes con el caso wichí (Gómez, 2009). Entre los wichí, el mito también pone en escena una representación específica del parentesco y de las relaciones matrimoniales. Esquemáticamente, el relato narra el destino de un hombre común que se enamora de una estrella, la cual vive junto a él en la tierra hasta que sufre un contratiempo y determina retornar al cielo. El esposo decide buscarla y en el cielo encuentra finalmente la muerte. El cielo representa el ámbito uxorilocal, lugar de residencia de la mujer estrella y su familia, con lo cual el relato escenifica la situación desventajosa del afín masculino en el hogar uxorilocal.

Por otra parte, «los estudios» transmitidos por «los antiguos» enseñan que Luna o A'woGoyk es un ser masculino que nace, crece y muere cíclicamente. Tal como ha reportado Métraux (1946b: 19), muchos grupos tobas consideran 
todavía que Luna es un ser humano (Blixen, 1992: 20-21). Los entrevistados lo indican constantemente: « $A^{\prime}$ 'woGoyk es un hombre (...) Nadie conoce el cielo: solo $A^{\prime}$ woGoyk», quien claramente es un ser masculino que puede ir y regresar del cielo. A diferencia de lo que ocurre en otras parcialidades tobas, no es que Luna 'mira a las mujeres' para que tengan su período (Tola, 2001), sino que baja a la tierra para tener relaciones con cada una de ellas. Este movimiento entre ambos planos es evidente para los tobas. La forma de Luna es leída junto a la trayectoria celeste que el astro describe a través del mes. En un primer momento intenté indagar sobre la luna utilizando gráficos de las formas lunares a medida que pasaba el mes (cuarto creciente, menguante, luna llena), pero fue poco efectivo, pues la forma toba de indicar cada uno de los ciclos de las fases lunares consiste en describir la porción visible de la luna mientras se indica con un brazo el trayecto y la posición celestes de la misma, en los diferentes momentos del día o de la noche. Este marco de referencia brinda los datos suficientes para conocer el momento del ciclo vital en el que se encuentra Luna. Si se la ve hacia el Oeste, apenas anochece, antes de desaparecer, Luna es «joven»: se la llama «luna tierna» o a 'woGoyk dalaichi, «muy pequeño» o ka'leka, kat'ñwk, «tierno» o 'dalagaik. La mayoría de los términos asociados con esta fase lunar refieren a la juventud7.

Pero a medida que pasa el tiempo, Luna comienza a madurar, asumiendo el nombre a'vik 'taraik, que significa «muy maduro» o bien taa'ñi, «duro». Para hablar sobre esta luna los tobas indican con el brazo el trayecto que hace durante toda la noche del Este hacia el Oeste. Muy distinta es la aparición de Luna anciano, tarde por la noche, que sigue su trayecto durante el día en estado pequeño y débil —así, cuando está en lo que nosotros Ilamaríamos «cuarto menguante», durante su tercer ciclo de fases, se llama a Luna yagai ${ }^{\prime} k i$ : «anciano» o «viejo»—. Luego de este ciclo, Luna desaparece: yilew («muerto»). Los tobas dicen que este personaje no es como waqachi'ñi (las estrellas), pues ellas no cumplen con las etapas del ciclo vital. Por otro lado, con la luna se describen períodos temporales más breves que las estaciones y más largos que los días, operando como una suerte de equivalente de nuestra idea de «mes». La luna, pese a ser un ser masculino, se liga estrechamente con la feminidad. A'woGoyk es así el encargado de «hacer» las mujeres, provocando la menarca o neta'gae. Como explicó una mujer toba: «es como si Luna me hubiera violado». De esta forma, las mujeres necesitan de una intermediación de carácter masculino para ser fehacientemente mujeres, casarse y poder tener hijos (Gómez, 2010). Durante los períodos posteriores, de hecho, la mujer sabe en qué «luna» tiene su período, y antiguamente, contando las lunas en las que no tienen su menstruación, las mujeres sabían si estaban embarazadas (Gómez, 2010).

Al contrario del caso de Luna, los tobas no se explayan demasiado sobre el sol o A'hewa. Simplemente explican que por medio del trayecto celeste, el sol da cuenta del transcurso del día, y que cada día se cuenta al amanecer. El equivalente a las horas también se marca por medio del sol. Hoy en día, los tobas ya utilizan

7 Se ha recogido otro término en el que se destaca la luna mirando hacia el Norte, zona tradicionalmente ligada a los nivaclés: en este contexto, se la llama teyaGaiki, que literalmente significa «mira hacia los nivaclés». 
el reloj, pero generalmente, al hablar sobre un momento del día, indican con su brazo extendido hacia el cielo el lugar exacto que ocupa el sol en ese momento. Las mitologías toba y pilagá coinciden en que el sol es poderoso; sin embargo, entre los tobas occidentales, el sol es un hombre, en tanto que es considerado mujer entre los pilagá (Wilbert \& Simoneau, 1989: 3-10). Un mito pilagá describe un ser femenino muy poderoso, que se transforma por sí mismo en mujer anciana o en una muchacha (Wilbert \& Simoneau, 1989: 3-4). Según lo reportado por Métraux (1946b:19; 1973: 129), el sol también puede aparecer bajo forma femenina andando lentamente en invierno porque es una anciana; y acelerando durante el verano porque es una jovencita.

Como Luna, Dapi 'chi es un ser masculino que también cumple con las exigencias inexorables del ciclo vital. Entre los tobas occidentales, Tomasini (1976) describía a este personaje como un «alto Dios uránico». Hoy en día, luego de la intensa labor evangelizadora, los tobas no dicen lo mismo y en ninguno de los testimonios aparece como «creador» o como «Dios». Sin embargo, se sigue considerando un personaje importante. Idoyaga Molina (1985: 80) transcribe un mito pilagá en el cual Dapi 'chi vivía en los límites de la tierra junto con los seres humanos, pero luego de sufrir agresiones por parte de ellos se retira al cielo y desde allí castiga a quienes lo habían ofendido, enviando una helada que bloqueó al sol, hasta que los hombres comenzaron a desesperar. Finalmente el sol volvió a aparecer, y entonces Dapi'chi revivió a parte de la humanidad. Eso explicaría el antiguo temor respecto de la posible desaparición del asterismo al comenzar el ciclo anual, que significaría la falta de prosperidad ese año. Dapi'chi tiene su origen mítico en la tierra y luego de cumplir sus tareas se retira al cielo. Se puede afirmar que este asterismo es el que tiene una mayor importancia cotidiana para los tobas. Se considera que Dapi'chi es joven, «nuevito», durante su amanecer helíaco, cuando reaparece luego de un tiempo de invisibilidad por el horizonte oriental, poco antes del amanecer (hay que considerar que el amanecer helíaco de las Pléyades es por el horizonte noreste). Para la cosmovisión toba, este fenómeno es fundamental. Esta aparición se da durante los primeros días de junio, durante naqabia 'Ga, la fase fría del ciclo anual. La relación entre Dapi'chi y el frío es crucial: justamente, como sugerimos, se trata del «dueño» o lo'got ${ }^{9}$ de las heladas y, por tanto, del encargado de administrarlas. Si antes del amanecer helíaco comienza a sentirse frío, se dice que Dapi 'chi ya está presente, por más que todavía no se lo pueda ver en el cielo nocturno. A medida que transcurren las madrugadas, con el correr de los meses, comienza a aparecer en el horizonte antes del amanecer. Dapi'chi es visto cada vez más alto en el cielo y cada vez más lejos del horizonte. Ese movimiento va indicando que día a día, mes a mes, el asterismo envejece y pierde vigor. Sigue enviando heladas, pero ya se van acercando las últimas porque poco

8 Datos recabados por Tomasini entre los tobas que la literatura chaqueña denomina «occidentales», situados en el paraje Monte Carmelo, al sureste del pueblo llamado Hito 1, en la provincia de Salta (Argentina).

9 Para los tobas, cada uno de los ámbitos del mundo (monte, río, llanura, etc.) tiene un «dueño» o «encargado» (lo 'got). Para entrar a un ámbito bajo la potestad de un «dueño», o bien para solicitar su intervención o benevolencia, los tobas aún se comunican con ellos mediante palabras ritualizadas. 
a poco va «muriendo» hasta finalmente «desaparecer». Cuando está en su punto más alto en el cielo al amanecer, significa que ya está viejo: yagai 'ki. Esta situación se da recién a principios de septiembre, en nawo 'Go. Luego Dapi 'chi entra en su ocaso cósmico, y aunque todavía puede ser visto cada mañana hacia el horizonte Oeste, se dice que «ya no está», y su importancia cosmológica se diluye. Esta posición menguante suele ir acompañada de un clima cambiante: ya no hace frío y poco a poco llega la etapa más próspera del año: 'wo'e (Gómez, 2011; 2012). En esa etapa del ciclo anual Las Pléyades pueden verse en el cielo apenas anochece, pero ya no asociadas con Dapi'chi. Dapi'chi propicia la época de fertilidad y abundancia por medio de las heladas, pero eso no implica que esté en el cielo cuando dicho período tiene lugar; en la óptica toba, de hecho, eso sería un contrasentido.

A nivel sociológico, puede decirse a la vez que Dapi 'chi mantiene relaciones estructurales con el asterismo formado por el Cinturón de Orión, llamado Qarko’tel o Neko’tel. Esta relación evoca la oposición simbólica entre lo masculino, representado por Dapi 'chi, y lo femenino, representado por Qarko 'tel. Dapi 'chi es considerado un haliaGa'nek (líder), guía para el resto de las estrellas. Por eso aparece primero y en su amanecer helíaco se lo percibe con una corona o noko'pa. Esa diadema de plumas ornamental caracterizaba al haliaGa'nek guerrero, pues solo aquel que portaba plumas rojas había logrado matar a un enemigo en combate. Por más que la figura del liderazgo sea compleja y debió ir cambiando a través de los años incorporando nuevos valores, el asterismo rememora de forma recurrente la antigua e idealizada figura del líder guerrero ${ }^{10}$ (Gómez, 2011; 2012).

Por su parte, el asterismo femenino asociado al Cinturón de Orión es Ilamado tanto Qarko'tel («nuestras abuelas») como Neko'tel («las abuelas de alguien»). A Qarko'tel se lo relaciona inmediatamente con Dapi'chi pues, en general, al nombrar a este último se entiende que detrás suyo aparece ese asterismo. Al contrario de Dapi'chi que, en un principio, es vigoroso y joven, Qarko'tel representa desde su aparición misma a unas «viejas», y su singularidad simbólica está definida por esa condición, que en el caso de Dapi'chi se adquiere con el paso del tiempo. Así, los tobas expresan con certeza que Qarko'tel son «las ancianas del cielo» mientras que el resto de las estrellas waqachi'ñi son mujeres jóvenes. Las primeras tienen a su cargo el cuidado y formación de las estrellas jóvenes - aquellas que, recordemos, han quedado en el cielo y no han bajado a la tierra (Gómez, 2010; 2012)—. Esta idea de transmisión de «los estudios» por parte de las abuelas a sus nietas es una norma ideal vigente entre los tobas. Por otro lado, aunque el Cinturón de Orión se asocia estrechamente con Dapi'chi, al contrario de lo que sucede con Dapi'chi, Qarko’tel (el asterismo representado por el Cinturón de Orión) es reconocido en todo momento en la bóveda celeste: en las peores épocas de frío (junio y julio) y también en los momentos

10 Respecto del liderazgo indígena chaqueño y sus cambios, ver los estudios en Braunstein \& Meichtry, eds., 2008. 
más calurosos (diciembre a enero). En una palabra, estas estrellas son en todo momento Qarko’tel, nuestras abuelas.

Parece plantearse, entonces, un contraste entre las estrellas jóvenes (waqachi 'ñi) o viejas (Qarko'tel), por el lado femenino, y entre Dapi'chi y A'woGoyk (Luna) por el lado masculino. Los seres masculinos y femeninos tienen una relación diferencial con respecto a la duración: A 'woGoyk y Dapi 'chi cumplen claramente con las etapas dinámicas del ciclo vital, mientras que waqachi'ñi y Qarko'tel mantienen en todo momento sus roles etarios.

\section{LA VÍA LÁCTEA, LOS VIENTOS Y LOS ESTRATOS CELESTES}

Hasta aquí hemos mencionado entidades que ninguna persona indígena o no indígena dudaría en clasificar como pertenecientes al ámbito celeste. Sin embargo para alguien que no es toba hay otros objetos y fenómenos que, en principio, no parecerían ligados con el cielo de forma tan directa, pero que en la perspectiva toba se asocian con él.

Al hablar del cielo los tobas difícilmente olvidan nombrar al Na 'qaik o al Ma'ñik. Al estar «dibujados» en la Vía Láctea, estos asterismos atraviesan el firmamento nocturno y nos permiten comprender su profundidad. Cuando se refieren a ellos, los describen como un camino, Na qaik, y también como un Ma'ñik (suri o ñandú, Rhea americana)11. Asimismo, la Vía Láctea aparece referida como Lakawa'hegem. En estos asterismos, puede percibirse una cierta propensión a la flexibilidad semántica ante los cambios socioculturales.

La gran mayoría de los tobas reconocen en el cielo el Ma'ñik12 uniendo en un mismo diseño varias zonas oscuras de la Vía Láctea13. Algunos interlocutores explican que el suri que se ve en el firmamento llegó hasta allí al escapar de unos perros que lo perseguían. Pero, sobre todo, explican que el Ma'ñik está en el «camino», Na qaik, y que las manchas blanquecinas que hay a su alrededor se deben a la polvareda que levanta al correr. Además, se entiende que flota en el cielo como «una señal» y se asocia la figura celeste con los cuidados cinegéticos que hay que tener al cazar suris. Tal como señalan Arenas \& Porini (2009), este ave es muy importante a nivel cosmológico y es por tanto una de las más representadas en los telares. Además, el suri se relaciona simbólicamente con cuestiones sociales

11 Para indagar sobre la Vía Láctea en otros grupos, cf. Giménez Benítez et al. (2002); López \& Giménez Benítez (2007); López (2009).

12 Para señalar al ma 'ñik celeste, un entrevistado nos comentaba que se dice ma'ñik (suri) di' 'me (el acostado/muerto/dormido). De esa forma, si se dice di' 'me ma'ñik, se sabe de inmediato que se hace referencia a aquella forma que se dibuja en el cielo.

13 Este asterismo es diseñado para que el «Saco de Carbón» forme la cabeza del Suri, en tanto que los manchones oscuros representan el cuerpo: así, tanto el cuello como el pecho del animal podrían describirse como «manchones negros» fácilmente visibles en la franja blanquecina que forma la Vía Láctea y que resultan muy cercanos a la constelación de Escorpio. El trazo celeste que los tobas occidentales realizan del ma ñik es pues distinto al de los «avestruces siderales» reportados por Lehmann-Nitsche (1923b: 276-278, 281). 
positivas: la domesticación, la alimentación, la medicina, la fabricación de bienes materiales. Asimismo, resulta interesante que se perciba al Ma'ñik trasladándose por el camino, $\mathrm{Na}^{\prime}$ 'qaik, puesto que es por medio del camino y de la hendidura que traza en el firmamento que puede percibirse el resto de los niveles del cielo («estrellas muy pequeñitas»), más lejanos o altos, donde solo los shamanes pioGo 'nak pueden llegar al comunicarse con los pa'yak («espíritus» que, luego de la labor de los misioneros anglicanos, pueden conceptuarse también como poderes «demoníacos»).

Los tobas conocen bien los movimientos de la Vía Láctea a medida que pasan las horas nocturnas y también los meses; de ahí que describan al Na'qaik

... como un nivel que va guiando y cuando se pone derechita donde sale el sol, es que va a levantar el sol (...) es un camino, se mueve y atraviesa. Se va doblando de este a oeste y va como de sureste a noreste: Algunos le dicen Lakawa'hegem.

Así, al mismo referente empírico al que los «antiguos» llamaban Na'qaik se añade otra denominación que recoge los significados del antiguo asterismo, pero adaptados a las nuevas interpretaciones ligadas con la hermenéutica bíblica. Esta nueva interpretación relaciona la oración cristiana del «padre nuestro» con los tradicionales niveles tobas del cielo. La oración comienza diciendo «padre nuestro que estás en los cielos», lo cual para ellos refiere a su visión tradicional del cosmos (Gómez, 2008). De esta forma, se interpreta que, por medio de Lakawa'hegem, se pueden divisar los tradicionales tres niveles celestes, que posibilitan la comunicación entre el cielo y la tierra. Según unos de los pastores anglicanos tobas, así justamente lo anuncia la propia Biblia: «un lugar que está partido como un río». Las descripciones tobas concuerdan en que en el medio de este espacio astral, poderoso y visible a través de la Vía Láctea, hay monstruos, remolinos, fuertes vientos y nieve. No extraña entonces que se entienda que a través de dicha hendidura bajará Jesús cuando llegue su hora, y se desate el temido Apocalipsis. Un hombre toba, de aproximadamente 30 años, explica así que el término Lakawa'hegem refiere a un espacio que está abierto, que va desde la tierra hacia arriba. Aunque el Génesis habla de una división de aguas «de arriba» $y$ «de abajo», igualmente lo asocian al término Lakawa 'hegem que parece referir más específicamente a un quiebre o hendidura del plano celeste ligada con el viento sureste-noreste. Si se segmenta el término, puede observarse que se relaciona con los vientos, y más específicamente con aquellos vientos que corren por puntos que podrían llamarse intercardinales: el nordeste y el sureste.

La'yat14 es la categoría genérica que identifica todos los vientos. Sin embargo, se llama waqia'gaik a aquel viento que sopla desde el Norte; se dice que es un viento muy fuerte y caluroso. A pesar de todos los inconvenientes que desencadena la llegada de waqia'gaik, es muy esperada. Quienes siembran dicen

${ }^{14}$ Sería interesante ampliar la investigación en el futuro, dilucidando los deícticos que determinan el género y la relación con el hablante de cada uno de los términos relacionados con el cielo, pero actualmente, en esta etapa de la investigación, no poseo la información necesaria para hacerlo. 
que su fuerza produce un movimiento que hace crecer rápido algunas plantas. Ese viento generalmente comienza a soplar durante agosto, cuando tiene lugar una nueva temporada del ciclo anual que los tobas llaman nawo 'Go. En ese período empiezan a florecer algunos árboles. Antes de nawo 'Go tiene lugar naqabia 'Ga, un corto lapso de frío intenso que sabemos asociado con Dapi'chi. Naqabia 'Ga (junio y julio) es una época de sequía, durante la cual el monte no ofrece frutos y sopla kola'waik, el viento Sur. El viento Sur también es descrito como un viento muy fuerte, pero frío. Los tobas dicen que, cuando comienza a soplar, es una de las señales de la pronta aparición de Dapi'chi (las Pléyades) y Qarko'tel (Tres Marías o Cinturón de Orión), indicando la inminencia de la estación naqabia 'Ga. La oposición entre estos vientos desmesurados parece brindarnos pistas para seguir indagando en el futuro la gran diferencia entre los momentos propicios del año, mayormente ligados al calor y la abundancia. Contrastan con los tiempos en los cuales prima el frío, cuando comienza la sequía, que se liga íntimamente a la carestía y a la falta total de alimentos en el monte y en los cuales el río Pilcomayo parece desvanecerse.

Finalmente, los tobas también nombran a laka'wa', un «viento bueno», suave, menos intenso, que puede venir tanto del Este como del Oeste: «como que va saltando». Un joven agrega una precisión: laka 'wa' «traba», contiene, modera o apacigua a los otros vientos más fuertes. Cuando los vientos más poderosos chocan con laka 'wa', este los frena; por eso el informante habla de su «bondad» al atemperar aquellos otros vientos catalogados como excesivamente poderosos. A pesar de que laka 'wa' sopla tanto desde el Este como desde el Oeste, cuando los tobas señalan con sus manos las direcciones por las cuales se mueve laka 'wa', marcan siempre puntos intercardinales, con un desplazamiento desde el Sur hacia el Norte por el lado oriental —esto es, en dirección Sureste-Noreste-

«Fuertes», «calurosos», «fríos», «cansados», «portadores de enfermedades», relacionados con «bichos» $\mathrm{u}$ «hombres endemoniados»: son algunas de las tantas caracterizaciones de los vientos waqia'gaik (viento norte), kola'waik (viento sur), nawoma'Ga (tormenta repentina) y kada'a'chi (torbellino). Pero a la vez hay un viento catalogado como suave, sereno, que viene desde los puntos intercardinales para contrapesar los vientos fuertes. En definitiva, parece constatarse una suerte de reflexión cosmológica que traduce en clave eólica cierta oposición moral entre el viento laka'wa', que encarna las virtudes de la moderación, lo auspicioso, la temperancia en todos sus sentidos, y los demás vientos, que simbolizan por su parte la desmesura, el exceso y la incontinencia —oposición, por otra parte, que los discursos misioneros anglicanos aprovechan naturalmente en una especie de dicotomía ética entre el «bien» y el «mal», entre «vientos buenos»y $\mathrm{y}$ «vientos malos»—-

Hemos visto al segmentar la palabra lakawa 'hegem (cuadro 1) que contiene una clara referencia al viento laka'wa'. Pero no es la única oportunidad en la cual se relacionan ambos fenómenos. Al leer detenidamente las descripciones del asterismo, se comprueba en efecto que refiere a un lugar semejante a un río, en el cual hay viento y nieve: un espacio celeste que se hunde hacia arriba, atravesado por una gran corriente en continuo movimiento. Otra de sus particularidades es 
Notas para una cartografía oral del cielo entre los tobas del oeste formoseño

Cuadro 1 - Segmentación del término en morfemas

\begin{tabular}{|l|l|l|}
\hline \multicolumn{2}{|l|}{ Lakawa'hegem } \\
\hline $\mathrm{L}$ & akawa & hegem \\
\hline 3POS & viento sureste - noreste & sufijo: «hacia arriba» \\
\hline \multicolumn{2}{|l}{ Su viento sureste-noreste hacia arriba } \\
\hline
\end{tabular}

el movimiento que realiza en el transcurso de la noche, a medida que pasan los meses, similar al itinerario que dibuja el viento llamado laka 'wa' por los puntos intercardinales: así, cada vez que la vía láctea —lakawa’hegem o na'qaikatraviesa el firmamento, va corriendo oblicuamente por el mismo eje Norte-Sur que marcan los vientos fuertes: waqia' gaik (viento Norte) y kola 'waik (viento Sur). El patrón de movimientos de este espacio poderoso es pues similar al descrito por el viento laka 'wa'. Uno de los ancianos, de hecho, comenta que laka 'wa' es un viento que pasa por el cielo y que na quik sería «la casa del viento». Otros tobas explican que Lakawa'hegem es $\mathrm{Na}^{\prime}$ qaik, un camino, y que el viento entra allí para no soplar fuerte. Así como Laka 'wa' es valorado como un «viento bueno», moderado, apaciguador, que atempera a sus pares dañinos, su contraparte celeste opera como una suerte de vía de comunicación privilegiada entre el cielo y la tierra. En la franja en la que se hunde Lakawa'hegem corre en efecto mucho viento; se dice que la misma se va moviendo gracias al viento. Se trata de un espacio partido, un cielo «abierto» como una hendidura surcada por vientos, que deja a la vista la existencia de otros planos celestes. Por medio de este asterismo, en suma, puede verse el más allá; por lo tanto, simbólicamente, posibilita la comunicación tanto con el Dios cristiano como con el resto de los «poderosos» que habitan el cielo15.

De esta forma, advertimos cómo cada una de las explicaciones brindadas sobre la Vía Láctea como Na 'qaik y Lakawa 'hegem se van complementando entre sí para llegar a dar una imagen comprensiva del cielo y de su permanente comunicación con los otros planos del universo. Por su lado, las lecturas celestes van variando

15 Esta idea guarda una cierta similitud con la representación del árbol o eje del mundo que comunica los diversos planos del cosmos. Testimonios del eje arbóreo fueron documentados en grupos de habla guaycurú, como por ejemplo los mocovíes (Lehmann-Nitsche, 1924a:76; 1927; López, 2009), los tobas y pilagá del río Pilcomayo (Métraux, 1946b: 24-26) o los tobas de Miraflores (Cordeu, 19691970:123-124). Anteriormente, el padre Guevara había reportado el árbol cósmico durante el siglo XVIII (Métraux, 1946b: 24) y lo mismo sucede entre los wichí (Métraux, 1946b: 24-25). Además, se sabe que los mocovíes hablan de la existencia de «túneles» o «caminos» que enlazan diferentes lugares del cosmos, significativamente «hechos de viento», que solo los shamanes pueden detectar (López, 2009: 219). Por su parte, Wright (2008: 152-153) analiza el árbol negro (nawe' 'epaq) y su relación con la Vía Láctea, y afirma que se asemeja «al árbol cósmico que funcionaba como canal de comunicación entre los diferentes niveles» y que son los shamanes quienes lo transitan oníricamente, guiados por los espíritus auxiliares. 
concomitantemente a los cambios socioculturales que acontecen en la vida de los tobas. De esta forma, pueden establecerse conexiones entre la visión más «tradicional» del cielo y la que se relaciona con la interpretación que se hace del mismo en clave bíblica: una hermenéutica que asocia los saberes tradicionales con las enseñanzas brindadas por el cristianismo anglicano.

\section{CONSIDERACIONES FINALES}

Podemos apreciar que el entretejido fluido de vientos y planos celestes remite no solo a una conexión entre los ámbitos del cosmos, sino a la vez a cierta adaptación plástica y flexible ante los cambios que generó la adopción del cristianismo anglicano. El frío también se asocia con el cielo, y hay momentos en los cuales esto repercute en la tierra. Esto es obvio para los tobas, puesto que para ellos el cielo, lugar frío por excelencia, está más próximo a la tierra durante el invierno. Prueba de lo anterior es que al cielo nocturno se lo aprecia mucho mejor y más claramente durante la época fría. De hecho, tanto la cercanía del cielo como la de Dapi 'chi son las que, junto con otros factores como el viento Sur, hacen que en la tierra se sienta el frío. Dapi 'chi es quien envía las heladas desde el cielo cuando está cerca de la tierra, en los amaneceres posteriores a su orto helíaco en el hemisferio sur, durante la estación Naqabia'Ga. Esta relación con el frío se ve corroborada porque cuando el cielo y Dapi'chi «se alejan», este último «envejece» y comienza a desvanecerse el frío; a medida que transcurren los meses, el asterismo parece ir alejándose del horizonte. Cuando Dapi 'chi llega a su punto máximo de altura respecto del horizonte, caen las últimas heladas. Durante ese lapso el frío comienza a desvanecerse y con él el poder del asterismo, que evidentemente ha envejecido. En ese instante ha llegado nawo 'Go y la presencia del viento Norte (waqia'gaik) decreta el final de la temporada fría. Ya en la época más calurosa del año, el cielo no se ve con claridad por estar «lejano» y Dapi 'chi ha desaparecido: aunque las Pléyades puedan verse durante la noche, ello no implica en modo alguno su presencia (Gómez, 2011). Aquí, también, la vejez se asocia con la falta de vigor para realizar las tareas cotidianas: pronto va a comenzar el momento más próspero del ciclo anual.

Dapi 'chi envía heladas cuando es joven y fuerte, lo cual es evidente en su colorida corona de plumas y por su proximidad con el horizonte. En ese momento es cuando más fuerza tiene y cuando más se siente el frío. Luna, en cambio, es «joven» cuando se la ve muy pequeña y durante el día hace su recorrido en el cielo desde el horizonte Este hacia el Oeste, hasta que al anochecer se la ve claramente cerca del horizonte occidental para luego desaparecer rápidamente. A medida que pasan las etapas, ambos asterismos cambian su forma y de posición en el cielo. Cada uno a su modo, Dapi 'chi y Luna cumplen con las exigencias del ciclo vital, lo cual parece ligarse con el hecho de ser personajes masculinos, asociados con la tierra y sus vicisitudes. De hecho, como se ha escrito, en la mitología de los grupos étnicos cercanos hay narraciones sobre el origen terrestre de ambos personajes que, por diversas circunstancias, deben migrar hacia el cielo (Tomasini, 
1976; Idoyaga Molina, 1985). Al no ser habitantes originarios del pi'yem, no sorprende que deban cumplir las etapas inexorables del ciclo vital: nacer, madurar, envejecer, extinguirse; la situación de los asterismos femeninos es bien distinta. Evocando acaso su origen celestial, Waqachi'ñi y Qarko'tel no cumplen el destino diacrónico de los seres vivos. Las Waqachi 'ñi permanecen eternamente jóvenes, y Qarko'tel remite en todo momento a las ideas de ancianidad, experiencia y sabiduría, las cuales se revelan en todo su esplendor a la hora de la menopausia, cuando las ancianas transmiten «los estudios» a las más jóvenes. A esto se suma que los asterismos femeninos son reconocidos como tales en cualquier momento del ciclo estacional. Todo parece indicar, pues, que las mujeres conservan algunos de sus atributos celestiales originarios: cierta peligrosidad, cierto poder, cierta situación liminal. Así como en el mito necesitan la participación del zorro para «domesticarlas», quebrando sus dientes vaginales, en la vida cotidiana esta mediación masculina se asocia con $A^{\prime}$ woGoyk (Luna). En la tierra las mujeres necesitan esa intermediación lunar masculina para cambiar y dejar de ser niñas, casarse y poder reproducirse. En el cielo, en cambio, la feminidad parece ser ontológicamente diversa: se es joven o se es anciana. Juventud y ancianidad no son estados vitales, sino una condición fija.

De algún modo, estos saberes constituyen «pequeños conocimientos» que, tal como ha señalado Lévi-Strauss (1979: 22), «han sido protegidos por su propia insignificancia». Muchas veces la belleza insondable del cielo, los misterios que parece engendrar y sus conexiones con ámbitos a los cuales solo pueden acceder cabalmente los pioGo'nak opacan pero tal vez protegen todo aquello que el firmamento nos dice sobre la sociedad que interpreta y es interpelada, día a día, noche a noche, por ese cielo.

\section{Agradecimientos}

Agradezco a Pastor Arenas y a Diego Villar por su colaboración.

\section{Referencias citadas}

ARÁOZ, G., 1884 - Navegación del Río Bermejo y Viajes al Gran Chaco, 416 pp.; Buenos Aires: Imprenta europea y taller de grabados en madera.

ARENAS, P., 1992-1993 - Fitonimia Toba-pilagá. Hacia una Nueva Carta Étnica, 5: 75-100. ARENAS, P., 2003 - Etnografía y Alimentación entre los Toba-Nachilamole\#ek y WichíLhuku'tas del Chaco Central (Argentina), 562 pp.; Buenos Aires: Dunken.

ARENAS, P., 2012 - Hechicería, muerte y prácticas funerarias para vengar maleficios entre los tobas del occidente de Formosa (Argentina). Una aproximación etnobiológica. In: Etnobotánica en zonas áridas y semiáridas del Cono Sur de Sudamérica (P. Arenas, ed.): 179-193; Buenos Aires: Centro de Estudios Farmacológicos y Botánicos. 
ARENAS, P. \& PORINI, G., 2009 - Las aves en la vida de los tobas del oeste de la provincia de Formosa (Argentina), 300 pp.; Asunción: Editorial Tiempo de Historia.

BARÚA, G. \& DASSO, M. C., 1999 - El papel femenino en la hostilidad wichí. In: Mito, guerra y venganza entre los wichí (M. Califano \& M. C. Dasso, eds.): 251-298; Buenos Aires: Ciudad Argentina.

BLIXEN, O., 1992 - Las manchas de la luna y sus explicaciones míticas en Sudamérica. MOANA. Estudios de Antropología, 4 (2): 5-65.

BOSSERT, F., BRAUNSTEIN, J. \& SIFFREDI, A., 2011 - A guerra interétnica no médio Pilcomayo (1883-1938). In: Os outros dos outros: relacões interétnicas na etnología (E. Coffasi da Lima \& L. Córdoba, eds.): 37-56; Curitiba: Editorial Universidade Federal do Parana (UFPR).

BRAUNSTEIN, J., 1988-1989 - Gentilicios toba del occidente chaqueño. Scripta ethnologica, 12: 51-55.

BRAUNSTEIN, J., 1989 - Astronomía mataca. Revisión crítica de la Mitología Sudamericana V de Roberto Lehmann-Nitsche. Scripta Ethnologica, Supplementa 9: 43-60.

BRAUNSTEIN, J., 1993 - Territorio e historia de los Narradores Matacos. Hacia una Nueva Carta Étnica del Gran Chaco, 5: 4-74.

BRAUNSTEIN, J. \& MEICHTRY, N. (eds.), 2008 - Liderazgo, representatividad y control social en el Gran Chaco, 312 pp.; Corrientes: Editorial Universitaria de la Universidad Nacional del Nordeste.

CARPIO, B., 2009 - Sistema de número en toba ñachilamole'k (Formosa, Argentina). Ponencia presentada en el $X$ Encuentro de Lingüística en el Noroeste; Sonora, México: Universidad de Sonora, Hermosillo, 12 al 15 de noviembre de 2008.

CARPIO, B., 2012 - Fonología y morfosintaxsis de la lengua hablada por los grupos tobas del oeste formoseño, 235 pp.; München: LINCOM. Europa Academic Publishers, n. 67.

COMBÈS, I., VILLAR, D. \& LOWREY, K., 2009 - Comparative Studies and the South American Gran Chaco. Tipití. Journal of the Society for the Anthropology of Lowland South america, 7 (1): 69-102.

CORDEU, E., 1969-1970 - Aproximación al horizonte mítico de los tobas, Runa, 12 (1-2): 67-176.

CORDEU, E., 1977 - Algunos personajes celestes de la mitología Ishir (Chamacoco). Suplemento Antropológico, Universidad Católica de Nuestra Señora de Asunción, 22 (1-2): 7-24.

CORDEU, E., 2000 - El Rayo y el Oso Hormiguero. La divinización de la lluvia en algunas mitologías chaqueñas. In: Actas del III Congreso Argentino de Americanistas: 119160; Buenos Aires: Sociedad Argentina de Americanistas.

DASSO, M. C., 1989 - Las transformaciones del sol y las estrellas en la cosmovisión de los mataco (grupo tachóhnai). Scripta Ethnologica, Supplementa 9: 19-42.

DOBRIZHOFFER, M., 1968 [1783] - Historia de los Abipones. Volumen II, 482 pp.; Resistencia: Universidad Nacional del Nordeste. Facultad de Humanidades, Departamento de Historia.

GIMÉNEZ BENÍTEZ, S., LÓPEZ, A. \& GRANADA, A., 2002 - Astronomía aborigen del Chaco: Mocovíes I. La noción de nayic (camino) como eje estructurador. Scripta Ethnologica, XXIII: 39-48.

GIMÉNEZ BENÍTEZ, S., LÓPEZ, A. \& GRANADA, A., 2006 - The sun and the moon as marks of time and spaces among the Mocovíes of the Argentinean Chaco. Archaeoastronomy, 20: 52-67; Austin: University of Texas Press. 
GÓMEZ, C., 2007 - Kates Ihukwetah. La representación social, material y estelar de la mujer entre los Wichí. Acta Americana, 15 (2): 97-116.

GÓMEZ, C., 2008 - Na'kaik, lakawa' hegem y dapi'chi. Huellas de una zona de frontera en dos representaciones celestes toba-pilagá. Estudios Latinoamericanos, 28: 185-209.

GÓMEZ, C., 2009 - Parentesco y representación estelar de la mujer entre los Wichí del Gran Chaco. In: Actas del VI ${ }^{\circ}$ Congreso de Americanistas: 205-219; Buenos Aires: Dunken.

GÓMEZ, C., 2010 - La luna y la feminidad entre los tobas del oeste formoseño (Gran Chaco, Argentina). Campos. Revista de Antropología Social, 11 (1): 47-64; Curitiba: Universidade Federal do Paraná.

GÓMEZ, C., 2011 - The Dapi 'chi (Pleiades) youth and old age: frost, air carnations and warriors. Cambridge Journals 7 I AU. S278 Archaeoastronomy and Ethnoastronomy. Building Bridges between Cultures): 50-57; Cambridge: Cambridge University Press, C.L.N. Ruggles ed.

GÓMEZ C., 2012 - El hombre y la mujer. Apuntes sobre la organización social toba y su relación con el ámbito celeste. Suplemento Antropológico, 47 (2): 7-112; Centro de Estudios Antropológicos.

GORDILLO, G., 2001 - «Un río tan salvaje e indómito como el indio toba»: una historia antropológica de la frontera del Pilcomayo. Desarrollo económico-Revista de Ciencias Sociales, 41 (162): 261-280.

GORDILlO, G., 2005 - Nosotros vamos a estar acá para siempre. Historias tobas, 222 pp.; Buenos Aires: Biblos.

IDOYAGA MOLINA, A., 1985 - Approach to the Pilagá culture of Central Chaco. Bulletin of the International Committee on Urgent Anthropological and Ethnological Research. Institut für Volkerkundë Universitätsstraße, 7, 27: 67- 86; Vienna I, Austria.

IDOYAGA MOLINA, A., 1986 - Estudio fenomenológico del mito de origen de las mujeres entre los pilagá (Chaco Central). Anales de arqueología y etnología, 41-42:165-174.

IDOYAGA MOLINA, A., 1989 - Astronomía pilagá. Scripta Ethnologica, Supplementa 9: 17-28.

IDOYAGA MOLINA, A., 2008 - El mito y las uniones imposibles. Análisis de la trama de un relato de los indígenas pilagá (Chaco Central). Revista Española de Antropología Americana, 38 (1): 91-104,

LEHMANN-NITSCHE, R., 1923a - La astronomía de los matacos. Revista del Museo de La Plata, 27: 253-265.

LEHMANN-NITSCHE, R., 1923b - La astronomía de los tobas. Revista del Museo de La Plata, 27: 267-285.

LEHMANN-NITSCHE, R., 1924a - La astronomía de los mocoví. Revista del Museo de La Plata, 28: 66-79.

LEHMANN-NITSCHE, R., 1924b - La astronomía de los vilela. Revista del Museo de La Plata, 28: 210-233.

LEHMANN-NITSCHE, R., 1924C - La astronomía de los chiriguanos. Revista del Museo de La Plata, 28: 80-102.

LEHMANN-NITSCHE, R., 1924-1925 - La astronomía de los tobas (segunda parte). Revista del Museo de La Plata, 28: 181-209.

LEHMANN-NITSCHE, R., 1927 - La astronomía de los mocoví (segunda parte). Revista del Museo de La Plata, 30: 145-159.

LÉVI-STRAUSS, C., 1979 - Introducción a la obra de Marcel Mauss. In: Marcel Mauss Sociología y Antropología: 13- 42; Madrid: Tecnos. 
LÓPEZ, A., 2009 - La Virgen, el Árbol y la Serpiente. Cielos e identidades en comunidades mocovíes del Chaco, 385 pp.; Buenos Aires: Universidad de Buenos Aires. Tesis Doctoral.

LOPEZ, A. \& GIMÉNEZ BENÍTEZ, S., 2007 - The Milky way and its structuring functions in the worldview of the Mocoví of Gran Chaco. Archaeologia Baltica, 10: 21-24.

LOZANO, P., 1989 [1733] - Descripción corográfica del Gran Chaco Gualamba, 466 pp.; Tucumán: Universidad Nacional del Tucumán, Departamento de Investigaciones Regionales, Publicación 288.

MENDOZA, M., 2002 - Band Mobility and Leadership among the Western Toba HunterGatherers of Gran Chaco in Argentina, vi + 233 pp.; Nueva York: Edwin Mellen.

MÉTRAUX, A., 1937 a - Études d'Ethnographie Toba-Pilaga. Anthropos, 32 (1-2): 171-194.

MÉTRAUX, A., 1937 b - Études d'Ethnographie Toba-Pilaga. Anthropos, 32 (3-4): 378-401.

MÉTRAUX, A., 1944 - Estudios de etnografía chaquense. Anales del Instituto de Etnografía Americana, 5: 263-314.

MÉTRAUX, A., 1946a - Ethnography of the Chaco. In: Handbook of South American Indians, vol. 1 (The Marginal tribes) (J. Steward, ed.): 197-370; Washington: Smithsonian Institution.

MÉTRAUX, A., 1946b - Myths of the Toba and Pilagá Indians of the Gran Chaco, 167 pp.; Philadelphia: American Folklore Society.

MÉTRAUX, A., 1973 - Religión y magias indígenas de América del Sur, xiii + 266 pp.; Madrid: Editorial Aguilar.

MILLER, E., 1975 - Shamans, Power Symbols, and Change in Argentine Toba culture. American Ethnologist, 2 (3): 477-496.

MILLER, E., 1977 - Simbolismo, conceptos de poder y cambio cultural de los tobas del Chaco argentino. In: Procesos de aculturación social (E. Hermitte \& Bartolomé, L., eds.): 305-338; Buenos Aires: Amorrortu.

NORDENSKIÖLD, E., 2002 [1912] - La vida de los indios. El Gran Chaco (Sudamérica), x + 312 pp.; La Paz: Ed. APCOB.

PALAVECINO, E., 1933 - Los indios pilagá del río Pilcomayo. Anales del Museo Nacional de Historia Natural, 37: 517-581; Buenos Aires.

PALAVECINO, E., 1969-1970 - Mitos de los indios tobas. Runa, 12 (1-2): 177-197.

PALMER, J. , 2005 - La buena voluntad wichí. Una espiritualidad indígena, 386 pp.; Formosa y Salta: Asociación para la Promoción de la Cultura y el Desarrollo.

PAUCKE, F., 2010 [1749-1767] - Hacia allá y para acá, 706 pp.; Santa Fe: Ministerio de Innovación y Cultura de la Provincia de Santa Fe, Argentina.

PÉREZ DIEZ, A., 1983 - A tale of the mataco about the origin of women. Latin American Indian Literatures, 7 (2): 134-169.

ROSTAGNO, E., 1969 [1911] - Informe. Fuerzas en operaciones en el Chaco 1911, 154 pp.; Buenos Aires: Círculo Militar.

RUIZ MORAS, E., 2000 - El rumor de los astros: una aproximación a la etnoastronomía toba taksek (Chaco Central). In: Actas del III Congreso Argentino de Americanistas, Volumen 2: 263-289; Buenos Aires: Sociedad Argentina de Americanistas.

STORM, O., 1892 - El río Pilcomayo y el Chaco boreal. Viajes y exploraciones, 151 pp.; Buenos Aires: Compañía sudamericana de billetes de banco.

TEBBOTH, T., 1943 - Diccionario Toba. Revista del Instituto de Antropología de la Universidad Nacional del Tucumán, 3 (2): 35-221.

TERÁN, B., 1998 - El cambio del año y el tiempo cíclico en la cosmovisión Mocoví. In: Actas del II Congreso Argentino de Americanistas, Volumen 1: 239-274; Buenos Aires: Sociedad Argentina de Americanistas. 
Notas para una cartografía oral del cielo entre los tobas del oeste formoseño

TERÁN, B., 2000 - Las medidas del tiempo y sus contextos culturales entre los toba orientales. In: Actas III Congreso Argentino de Americanistas, Volumen 2: 363-383; Buenos Aires: Sociedad Argentina de Americanistas.

TOLA, F., 2001 - La luna en el imaginario masculino y femenino de los tobas (qom) orientales del Chaco argentino. Avā Revista de Antropología, 3: 75-88.

TOMASINI, A., 1976 - Dapitchi, un alto Dios uránico de los Toba. Scripta Ethnologica, 4 (1): 69-87.

WILBERT, J. \& SIMONEAU, K. (eds.), 1982a - Folk Literature of the Mataco Indians, xvii + 507 pp.; Los Angeles: UCLA.

WILBERT, J. \& SIMONEAU, K. (eds.), 1982b - Folk Literature of the Toba Indians 1, 597 pp.; Los Angeles: UCLA.

WILBERT, J. \& SIMONEAU, K. (eds.), 1985 - Folk Literature of the Chorote Indians, 400 pp.; Los Angeles: UCLA.

WILBERT, J. \& SIMONEAU, K. (eds.), 1989 - Folk Literature of the Toba Indians 2, 597 pp.; Los Angeles: UCLA.

WRIGHT, P. , 2008 - Ser-en-el-sueño: crónicas de historia y vida toba, 270 pp.; Buenos Aires: Biblos. 